Journal of Physics and Its Applications

Journal homepage : https://ejournal2.undip.ac.id/index.php/jpa/index

\title{
The electric susceptibility of bi-layers ferroelectrics
}

\author{
Vincensius Gunawan¹, Ngurah Ayu Ketut Umiati², and Agus Subagio ${ }^{3}$
}

1Physics Department, Diponegoro University, Indonesia; goenangie@gmail.com

2Physics Department, Diponegoro University, Indonesia; ngurahayuketutumiati@gmail.com

3Physics Department, Diponegoro University, Indonesia; agussubagio@lecturer.undip.ac.id

\section{A R T I C L E I N F O}

\section{Article history:}

Received : 14 May 2019

Accepted : 2 June 2019

Available online : 12 June 2019

\section{Keywords:}

Electric Susceptibility

Polarization

Bilayer Ferroelectrics

\begin{abstract}
A B S T R A C T
In order to enhance insight of layered structure, we perform numerical calculation to obtain the dynamic electric susceptibility in bi-layers ferroelectrics. Since susceptibility is a parameter which gives response to the external field, then determination of this parameter is important. A lattice model is employed to slice bilayer structure into several lattices. Then, Landau-Khalatnikov equation of motion is used in each lattice to construct a matrix equation of equation of motion. The solution is obtained by applying entire-cell effective medium. We find that the homogeneity of dynamic polarization is different from homogeneity of the single individual layer due to the existence of interlayer interaction. As a result, the electric susceptibility is also altered. It is also noticed that there is a relation between the homogeneity of dynamic polarization and the value of electric susceptibility near resonant frequency. The higher the homogeneity, the bigger the values of susceptibility will be.
\end{abstract}

\section{Introduction}

Ferroelectric is defined as a dielectric which has an electric polarization without the existence of an external electric field. The popular example of this kind of material is $\mathrm{BaTiO}_{3}$. Since this material has potential application such as ferroelectric random access memory (FE-RAM) [1], polarization based adjustable memory [2], etc.,ferroelectric has been studied extensively for more than one decade both experimentally and theoretically.In the last decade, the study of ferroelectric involving many structures such as thin film [3-4], multilayers [5-6] and superlattices [7-8] structures had been performed due to its applications. These studies had motivated to control parameters for required applications. The multilayer and superlattice structures are hoped to possess better functional behaviours and properties compare to the parameters from the single individual layer. Since the functional application of the ferroelectric is predicted in the form of multilayer and superlattice structures, the study of the ferroelectric in the shape of layered structure become important.

The theoretical study of layered structure can be done using lattice model $[3,7,9]$. In this approach, material structure is divided into several lattices. This model is appropriate to handle internal interaction exists in the material, such as interaction between lattice polarization, depolarization at the surface, etc. This lattice model is also successfully used in the study of multiferroicheterostructure when the interaction at the surface between two materials is involved $[6,8,10]$. Since it is difficult to calculate the inhomogeneous parameters analytically, this model is suitable in calculating those parameters numerically (for example: the electric polarization in thin film ferroelectric).

Motivated to get better understanding of the behaviour of dynamic parameters in layered structure, we perform a study of the dynamic electric susceptibility in layered structure. To simplify the problem, we use bilayer with interaction between two different ferroelectrics existsat the interface instead of multilayer structure. Using LandauKhalatnikov (LK) equation of motion in lattice model of bilayer, the matrix equation of LK equation of motion is created. Then, the solution is obtained by performing numerical calculation using entire-cell effective medium approach which is successfully used in magnetic system [11] and multiferroics $[6,12]$.

\section{Research Method}

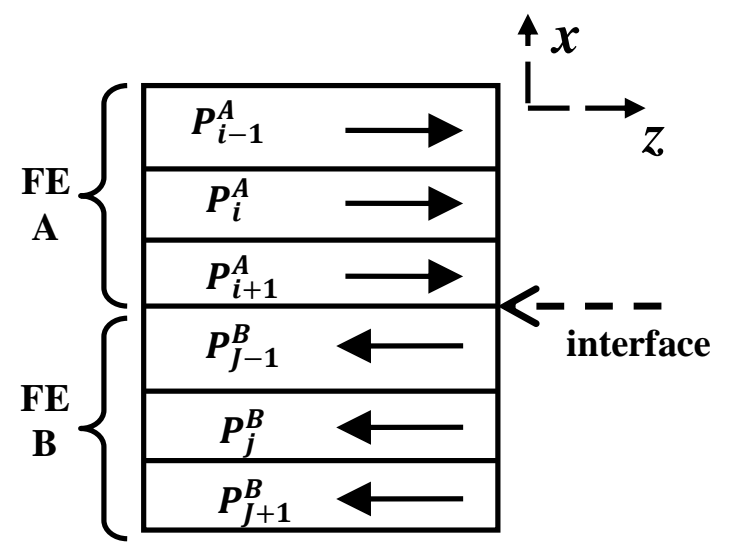

Fig.1: The illustration of the lattices model in this study. 
The geometry of the study is illustrated in Fig.1. The surfaces of top and bottom of the bilayer lieat $y$ $z$ plane. A bilayer of ferroelectric is comprised of two ferroelectrics. Ferroelectric A (FE A) with electric polarizations $P_{i}^{A}$ are parallel to $z$ direction is located above ferroelectric B (FE B) with polarizations $P_{j}^{B}$ are directed to the opposite direction of polarization in FE A (see Fig.1). Each of ferroelectric comprises of several lattices. In Fig.1, FE A consist of three lattices while $\mathrm{FE} B$ also contains three lattices. The interface between $\mathrm{FE} \mathrm{A}$ and $\mathrm{FE} \mathrm{B}$ is denoted by dashed arrow. Lattices are numbered from top to the bottom of the bilayer. Based on Fig.1, lattices 1, 2 and 3 are in the FE A. Lattice 4 is assumed to be interface while lattices 5, 6 and 7 are located in FE B.

We begin the calculation by determining the energy density of the bi-layers system as:

$$
F=F_{E}^{A}+F_{E}^{B}+F_{\text {int }}
$$

where $F_{E}^{A}$ and $F_{E}^{B}$ represent energy density of the first and the second ferroelectric. The energy density of the interface between those two ferroelectrics is denoted as $F_{\text {int }}$. Employing Landau theory of ferroelectric, the energy density of of the first ferroelectric (ferroelectric A ) can be written as fourth order polynomial series in form

$$
\begin{aligned}
& F_{E}^{A}=\sum_{i=1}^{n}\left\{\frac{a_{0}}{2}\left[T-T_{C}^{A}\right]\left(P_{i}^{A}\right)^{2}+\frac{\beta}{4}\left(P_{i}^{A}\right)^{4}\right. \\
& \left.+\frac{K_{A}}{2}\left[P_{i}^{A}-P_{i-1}^{A}\right]^{2}-E_{i}^{A} P_{i}^{A}\right\}
\end{aligned}
$$

where $a_{0}$ and $\beta$ represent Landau constant while $K_{A}$ is inter-lattices constant. Parameter $T_{C}^{A}$ is Curie temperature for FE A while $E_{i}^{A}$ and $P_{i}^{A}$ are electric field and electric polarization for lattice $i$ in ferroelectric A. The energy density for the second ferroelectric (ferroelectric B) is considered as

$$
\begin{aligned}
& F_{E}^{B}=\sum_{j=n+1}^{m}\left\{\frac{A_{0}}{2}\left[T-T_{C}^{B}\right]\left(P_{j}^{B}\right)^{2}+\frac{b}{4}\left(P_{j}^{B}\right)^{4}\right. \\
& \left.+\frac{K_{B}}{2}\left[P_{j}^{B}-P_{i-1}^{B}\right]^{2}-E_{j}^{B} P_{j}^{B}\right\} .
\end{aligned}
$$

Here, parameters $A_{0}$ and $b$ are Landau constant while inter-lattice constant is denoted as $K_{B}$. The Curie temperature for ferroelectric $B$ is represented by $T_{C}^{B}$. The electric field and polarization for lattice $j$ in ferroelectric B are symbolized by $E_{j}^{B}$ and $P_{j}^{B}$. The energy density from interface interaction between layer A and B which are located nearest to the interface is determined as

$F_{\text {int }}=\frac{\xi}{2}\left[P_{i}^{A}(i n t)-P_{j}^{B}(i n t)\right]^{2}$

where $\xi$ represents interaction constant between FE $\mathrm{A}$ and FE $\mathrm{B}$ and $P($ int $)$ denotes polarization at the lattice near interface. For example, based on the Fig. $1, P_{i}^{A}$ (int) $=P_{3}^{A}$ while $P_{j}^{B}($ int $)=P_{5}^{B}$.In this lattice model, the thickness of the lattice does not include in the expression of density energy as it is written in Eqs.(1)-(4) above.

In order to present dimensionless form of density energy, we used $P_{i}^{A}=P_{S}^{A} p_{i}^{A}$ with $P_{S}^{A}=\left(a_{0} T_{C}^{A} / \beta\right)^{1 / 2}$ and also $P_{j}^{B}=P_{S}^{B} p_{j}^{B}$ with $P_{S}^{B}=\left(A_{0} T_{C}^{B} / b\right)^{1 / 2}$ in Eq.(2) to Eq.(4). After that, we divide all terms in Eq.(1) with $\left(a_{0} T_{C}^{A}\right)^{2} / \beta$ resulting in dimensionless energy density

$f=f_{E}^{A}+f_{E}^{B}+f_{\text {int }}$

where

$$
\begin{gathered}
f_{E}^{A}=\sum_{i=1}^{n}\left\{\frac{1}{2}\left(\frac{T}{T_{C}^{A}}-1\right)\left(p_{i}^{A}\right)^{2}+\frac{1}{4}\left(p_{i}^{A}\right)^{4}\right. \\
\left.+\frac{\Gamma_{A}}{2}\left(p_{i}^{A}-p_{i-1}^{A}\right)^{2}-\tilde{E}_{i}^{A} p_{i}^{A}\right\} \\
f_{E}^{B}=\sum_{j=n+1}^{m} \alpha_{r} \beta_{r}\left\{\frac{1}{2} \alpha_{r}\left(\frac{T}{T_{C}^{B}}-1\right)\left(p_{j}^{B}\right)^{2}\right. \\
+\frac{1}{4} \alpha_{r}\left(p_{j}^{B}\right)^{4} \\
\left.+\frac{\Gamma_{B}}{2}\left(p_{j}^{B}-p_{j-1}^{B}\right)^{2}-\left(\alpha_{r} \beta_{r}\right)^{-1 / 2} \tilde{E}_{j}^{B} p_{j}^{B}\right\} \\
\text { and } \\
f_{\text {int }}=\frac{1}{2} \lambda\left[p_{i}^{A}(\text { int })-\left(\alpha_{r} \beta_{r}\right)^{1 / 2} p_{j}^{B}(\text { int })\right]^{2}
\end{gathered}
$$

Here, $\Gamma_{A}=K_{A} /\left(a_{0} T_{C}^{A}\right)$ and $\Gamma_{B}=K_{B} /\left(a_{0} T_{C}^{A}\right)$ while $\lambda=\left[\frac{\beta}{a_{0}^{3}\left(T_{C}^{A}\right)^{3}}\right]^{1 / 2} \xi$. Parameters Landau constant $\alpha_{r}$ and $\beta_{r}$ are defined as, $\alpha_{r}=\frac{A_{0} T_{C}^{B}}{a_{0} T_{C}^{A}}$ and $\quad \beta_{r}=\frac{\beta}{b}$. The electric fieldbecomes $\widetilde{E}_{i}^{A, B}=\left[\frac{\beta}{a_{0}^{3}\left(T_{C}^{A}\right)^{3}}\right]^{1 / 2} E_{i}^{A, B}$.

Then, minimizing the energy density respect to polarization, i.e: $\frac{d f}{d p}=0$, the equations (6) and (7) can be brought into the form as

$$
\begin{aligned}
& \quad\left[\left(\frac{T}{T_{C}^{A}}-1\right)+\Gamma_{A}\right] p_{i}^{A}+\left(p_{i}^{A}\right)^{3}-\Gamma_{A} p_{i-1}^{A}+\lambda p_{i}^{A}(\text { int }) \\
& -\lambda\left(\alpha_{r} \beta_{r}\right)^{\frac{1}{2}} p_{j}^{B}(\text { int })=0
\end{aligned}
$$

and

$$
\begin{gathered}
\left(\alpha_{r} \beta_{r}\right)\left[\alpha_{r}\left(\frac{T}{T_{C}^{B}}-1\right)+\Gamma_{B}\right] p_{j}^{B}+\alpha_{r}\left(p_{j}^{B}\right)^{3}-\Gamma_{B} p_{j-1}^{B} \\
+\lambda\left(\alpha_{r} \beta_{r}\right) p_{j}^{B}(\text { int })-\lambda\left(\alpha_{r} \beta_{r}\right)^{\frac{1}{2}} p_{i}^{A}(\text { int })=0 .
\end{gathered}
$$

By solving Eqs.(9) and (10) simultaneously, the dimensionless static polarizations $p_{i}^{A}$ and $p_{j}^{B}$ can be obtained. We then used those static polarizations in calculating the susceptibility of bilayer.

The susceptibility of the bilayer was calculated numerically using Landau-Khalatnikov Equation of motion as

$\frac{d^{2} P_{A, B}}{d t^{2}}=-f_{A, B} \frac{d F_{A, B}}{d P_{A, B}}$

wherefis inverse phonon mode. We assumed that the polarizations are comprised of static and dynamic polarization, $P_{A, B}=P_{A, B}^{S}+P_{A, B}^{d}$, where superscript $\mathrm{s}$ and $\mathrm{d}$ denote static and dynamic parts. The values of dynamic part are much smaller than the values of static polarization with the relation to time is written as $P_{A, B}^{d} \propto \exp \left(i \omega_{A, B} t\right)$ where $\omega_{A, B}$ 
represents a resonant frequency of ferroelectric $A$ or B. Then, by only considering the linear relation between the dynamic polarization and dynamic electric fields, we can write L-K equation of motion as

$\sum_{i=1}^{n}\left\{\left[\left(\omega_{i}^{A}\right)^{2}-\omega^{2}\right] P_{i}^{d, A}-K_{A} f_{A} P_{i}^{d, A}=f_{A} \varepsilon_{i}^{A}\right\}$

for lattices in ferroelectric A. Here, $P_{i}^{d, A}$ represents the dynamic polarization while $\varepsilon_{i}^{A}$ is dynamic electric field for lattices $i$ in ferroelectric $A$. The frequency in Eq.(12) is defined as

$\omega_{i}^{A}=\left\{f_{A}\left[a_{0} T_{c}^{A}\left(\frac{T}{T_{c}^{A}}-1\right)+3 \beta\left(P_{i}^{S, A}\right)^{2} K_{A}\right]\right\}^{1 / 2}(13)$

where $P_{i}^{S, A}$ is static polarization in lattice $i$ at ferroelectric A. Similarly, the equation of motion in ferroelectric B is written as

$\sum_{j=n+1}^{m}\left\{\left[\left(\omega_{j}^{B}\right)^{2}-\omega^{2}\right] P_{j}^{d, B}-K_{A} f_{A} P_{j}^{d, B}=f_{B} \varepsilon_{j}^{B}\right\}(14)$

where $P_{j}^{s, B}$ represents static polarization of layer $j$ at ferroelectric $B$ while resonant frequency is determined as

$\omega_{j}^{B}=\left\{f_{B}\left[A_{0} T_{c}^{B}\left(\frac{T}{T_{c}^{B}}-1\right)+3 b\left(P_{i}^{S, B}\right)^{2} K_{B}\right]\right\}^{1 / 2}$.

For the layer nearest the interface, the equation of motion can be easily derived by changing the constant $K_{A}$ or $K_{B}$ in Eq.(12) or Eq.(14) with $\lambda$, it yields

$\left[\omega_{A}^{2}\right.$ (int) $\left.-\omega^{2}\right] P_{A}^{d}($ int $)-\lambda f_{A} P_{B}^{d}($ int $)=f_{A} \varepsilon_{A}$ (int)

fromferroelectric A, and

$$
\left.\left[\omega_{B}^{2} \text { (int }\right)-\omega^{2}\right] P_{B}^{d}(\text { int })-\lambda f_{B} P_{A}^{d}(\text { int })=f_{B} \varepsilon_{B} \text { (int) }
$$

from ferroelectric B.

In order to solve this system of coupled equations, we employ entire-cell effective medium. This method requires consideration of the field's continuation at each layer. According to Maxwell's boundary condition, the continuity condition for tangential component of electric fields yields

$$
\varepsilon_{1}^{A}=\varepsilon_{2}^{A}=\cdots=\varepsilon_{A} \text { (int) }=\varepsilon_{B} \text { (int) }
$$$$
=\cdots=\varepsilon_{n+1}^{B}=\varepsilon_{m}^{B}=C_{z}
$$

where $C_{z}$ is a constant. By setting $C_{z}$, we then solve the equation of motion to obtain layer dynamic polarization $P_{i}^{d}$. The susceptibility of the bilayer is calculated using relation between the average polarization and electric fields as

$\left\langle P_{i}^{d}\right\rangle=\chi_{e}\left\langle\varepsilon_{i}\right\rangle$

The graphic of susceptibility is obtained by varying the frequency in a certain frequency interval.

\section{Result and Discussion}

In performing numerical study, we require parameters which represent ferroelectric $\mathrm{A}$ and ferroelectric B. For ferroelectric A, we used Curie temperature as $T_{C}^{A}=391 \mathrm{~K}$, Landau constant as $a_{0}=6.65 \times 10^{5} \mathrm{Vm} / \mathrm{C}^{-1} \mathrm{~K}^{-1}$ and the parameter $\beta=$ $3.56 \times 10^{9} \mathrm{Vm}^{5} / \mathrm{C}^{3}$. Also, the interaction constant $K_{A}=4.51 \times 10^{-9} \mathrm{Vm}^{3} / \mathrm{C}$. Theparameters above are appropriate for illustrating ferroelectric $\mathrm{BaTiO}_{3}$. Since we want that the resonance frequency of ferroelectric B is not far away from resonance frequency of ferroelectric A to simplify the analysis, then we set the parameters for ferroelectric B which have the same order as the parameters in ferroelectric A. The Curie temperature is set at $T_{C}^{B}=450 \mathrm{~K}$. The Landau constants are arranged at $A_{0}=2.65 \times 10^{5} \mathrm{Vm} / \mathrm{C}^{-1} \mathrm{~K}^{-1}$ and $b=1.56 \times$ $10^{9} \mathrm{Vm}^{5} / \mathrm{C}^{3}$. The interaction constant is adjusted as $K_{B}=0.51 \times 10^{-9} \mathrm{Vm}^{3} / \mathrm{C}$.

The static polarizations are calculated with the results are presented in Figure (2).
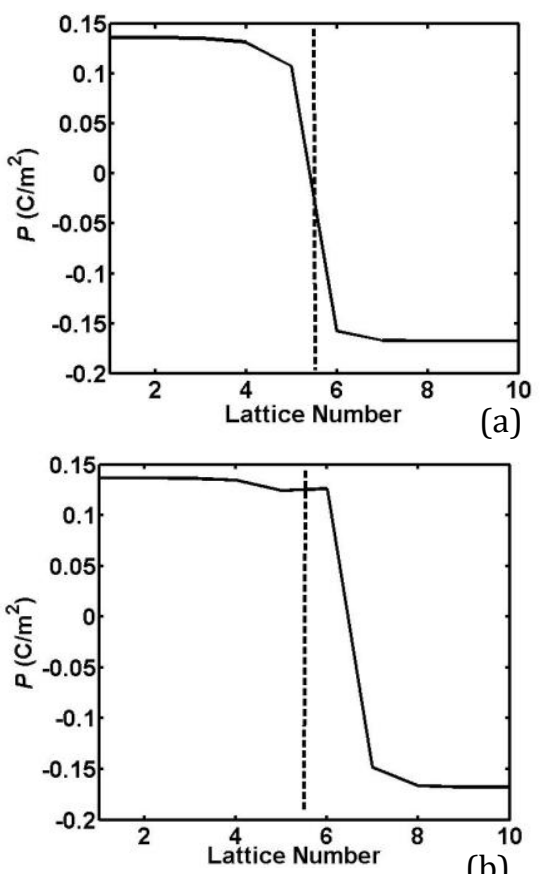

Fig. 2: Static polarization of bilayer ferroelectrics. (a) without interaction between ferroelectric $\mathrm{A}$ and $\mathrm{B}$.

(b) with interaction constant $\lambda=1 \times 10^{9} \mathrm{Vm}^{3} / C$.

Numerical calculation which is performed to obtain static spontaneous polarization is based on Eqs.(9) and (10). The results of numerical calculation are presented in Fig.(2). In Fig.(2), the vertical dashed lines represent interface. The left side ofinterface is ferroelectric A while the right side is ferroelectric B. Here, the number of lattices is set to five for each ferroelectric. We give numbers to the lattice as follow: lattice number 1 to 5 are lattices at ferroelectric $A$, while lattice 6 to 10 are lattices at ferroelectric B. Two conditions are considered. First, the circumstance without interaction between ferroelectric A and B $(\lambda=0)$. Second, the existence of the interaction between those two ferroelectrics is considered by setting interaction constant as $\lambda=1 \times 10^{9} \mathrm{Vm}^{3} / \mathrm{C}$. As it is illustrated in Fig. (2a), the first condition yields the discontinuity of the electric susceptibility at the region around interface. Without the interaction constant between ferroelectric A and B, each ferroelectric does not affect each other. Hence, their polarizations tend to have different values near the interface. The lattice 
polarizations in those two ferroelectrics affect each other when the interaction constant exists. This constant tends to decrease discontinuation of the electric polarizations at the interface. The lattice polarizations from ferroelectric A and ferroelectric B tend to have the same values near the interface, as it is shown in Fig.(2b).

These findings have similar trends with previous research in Ref.[8] which discuss the polarizations in the bilayer comprised of ferroelectric and multiferroic with the interaction between ferroelectric material and ferroelectric part of multiferroic exist.

The electric susceptibility of a bilayer ferroelectric is calculated numerically based on Eqs.(12) and (14). It is clear from those equations that the calculation process requires the values of static polarization from previous calculation. The results of the numerical calculation are expressed graphically in Fig. (3). In this picture, the resonant frequency near $\omega=32.3 \mathrm{~cm}^{-1}$ is possessed by ferroelectric B while frequency near $\omega=42 \mathrm{~cm}^{-1}$ is resonant frequency for ferroelectric A. Without interaction between ferroelectrics at the interface $(\lambda=0)$, the resulted susceptibility of the bilayer are just two independent susceptibilities which are drawn in one axis (see Fig.(3a)). From Fig.(2a), it can be seen that static polarization of ferroelectric $B$ is more uniform compare to the uniformity of ferroelectric A. As a consequence, the differences between static polarization at each lattice to the average polarization at ferroelectric B are smaller compare to the polarization differences in ferroelectric A. Hence, the resonant in ferroelectric $B$ is stronger than resonant in ferroelectric A. This is reflected by the values of susceptibility near resonant frequency, where the susceptibility of ferroelectric B is higher than the susceptibility of ferroelectric A.
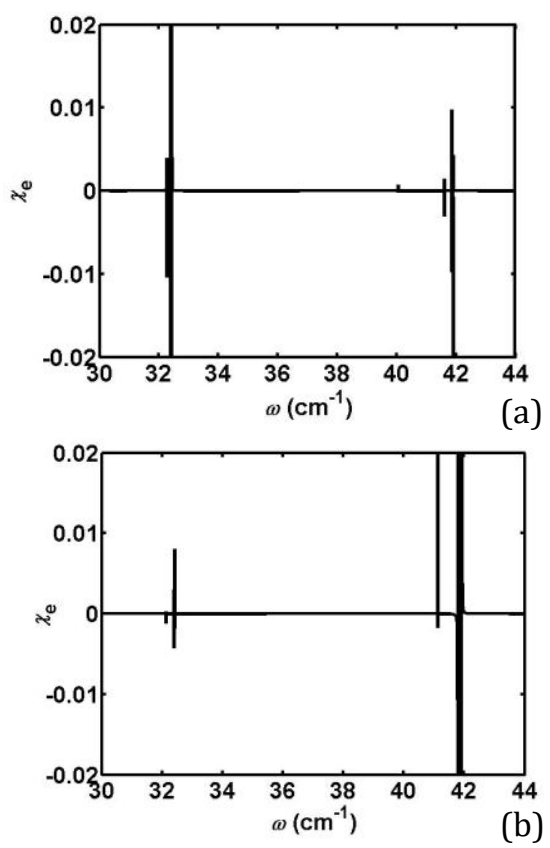

Fig. 3: The electric susceptibility of bilayer.

(a) without interaction between ferroelectrics.

(b) with interaction constant $\lambda=1 \times 10^{9} \mathrm{Vm}^{3} / \mathrm{C}$.
The electric susceptibility of a bilayer with interaction between ferroelectrics exists at the interface is shown in Fig.(3b). Since there is a change in discontinuity at the interface (see Fig.(2)) which affects the uniformity of the static polarization for both ferroelectric A and B, the susceptibility also changes. In this circumstance, the uniformity of spontaneous polarization at ferroelectric $\mathrm{A}$ is higher than that at ferroelectric B. Hence, the values of susceptibility near resonant frequency for ferroelectric $\mathrm{A}$ are higher compare to the susceptibility at ferroelectric B. It means that the resonance in ferroelectric $\mathrm{A}$ is stronger than the resonance of ferroelectric B. As an additional note, the resonant frequencies in bilayer ferroelectrics are similar to the resonant frequency of its ferroelectric components.

In this geometry, the value of $C_{z}$ is constant. According to Eq.(18), it means that the values of the lattice dynamic field are uniform. Hence, the properties of dynamic polarization are similar to the electric susceptibility. The dynamic polarizations also show resonant condition at certain frequency

By zooming the Fig.(3), we notice that the interaction constant changes the static polarization especially near interface (see Fig.2), which is in turn shift the resonance frequency. However, the shift is very small and difficult to be observed. The changes are also amplify or reduce the value of the electric susceptibility around the resonant frequency

\section{Conclusion}

In bilayer ferroelectric with an interaction at the interface, the polarization at the interface of ferroelectric $A$ and polarization at the interface of ferroelectric $B$ affect each other to decrease the polarization discontinuity at the interface. This process changes the uniformity of polarizations of the independent ferroelectric components. As a consequence, the electric susceptibility of bilayer is different from the susceptibility of its ferroelectric components

\section{Acknowledgment}

We acknowledge Faculty of Sciences and Mathematics, Diponegoro University for supporting this research through DIPA PNBP 2018 with contract No: 1756 1/UN7.5.8/PG/2018.

\section{References}

1. J. F. Scott, "Applications of modern ferroelectrics”, Science 315, 954 (2007).

2. T. Granzow, Th. Woike, M. Wohlecke, M. Imlau and W. Kleemann, "Polarization-based adjustable memory behavior in relaxor ferroelectrics", Physics Rev. Lett, 89, 127601 (2002).

3. L. Baudry and J. Tournier, "Lattice model for ferroelectric film materials including surface effects: Investigation on the depolarizing field properties," .J. Appl. Phys.90,1442 (2001).

4. K. H. Chew, L. H. Ong, J. Osman and D. R. Tilley, "Theory of far-infrared reflection and transmission by ferroelectric thin film," J. Opt. Soc. Am. B.18,1512 (2001). 
5. M. B. Okatan, J. V. Mantese and S.P. Alpay, "Polarization coupling in ferroelectric multilayers," Phys. Rev. B. 79, 174113 (2009).

6. V. Gunawan and R Stamps, "Ferromagnetic resonance shifts from electric fields: Field-enhanced screening charge in ferromagnet/ferroelectric multi-layers," Phys. Rev.B 85, 104411 (2012).

7. K. H. Chew, Y. Ishibashi and F. G. Shin, "A Lattice model for ferroelectric superlattices," Journal of the Physical Society Japan 75, 064712 (2006).

8. L. H. Ong and K. H. Chew, "Intermixing and magnetoelectric coupling in ferroelectric/multiferroicsuperlattices," Ferroelectrics 450, 7 (2013).

9. Y. Ishibashi, "A model of polarization reversal in ferroelectrics", Journal of the Physical Society Japan 59, 4148 (1990).
10. J. Zhan, R. C. Yin, T. Fan, M. H. Lu, Y. F. Chen, Y. Y. Zhu, S. N. Zhu and N. B. Ming, "Coupled phonon polaritons in a piezoelectric-piezomagnetic superlattice”, Phys. Rev. B 77, 075126 (2008).

11. R. L. Stamps and R. E. Camley, "Spin waves in antiferromagnetic thin films and multilayers: surface and interface exchange and entire-cell effective-medium theory", Phys. Rev. B 54,15200 (1996).

12. K. L. Livesey and R. L. Stamps, "High frequency susceptibility of a weak ferromagnet with magne-to strictive magnetoelectric coupling: Using hetero-structures to tailor electromagnon frequencies", Phys. Rev. B 81, 094405 (2010). 